\title{
Towards the map of quantum gravity
}

\author{
Jakub Mielczarek $^{1}$ • Tomasz Trześniewski ${ }^{1,2}$
}

Received: 13 December 2017 / Accepted: 13 May 2018 / Published online: 24 May 2018

(C) The Author(s) 2018

\begin{abstract}
In this paper we point out some possible links between different approaches to quantum gravity and theories of the Planck scale physics. In particular, connections between loop quantum gravity, causal dynamical triangulations, Hořava-Lifshitz gravity, asymptotic safety scenario, Quantum Graphity, deformations of relativistic symmetries and nonlinear phase space models are discussed. The main focus is on quantum deformations of the Hypersurface Deformations Algebra and Poincaré algebra, nonlinear structure of phase space, the running dimension of spacetime and nontrivial phase diagram of quantum gravity. We present an attempt to arrange the observed relations in the form of a graph, highlighting different aspects of quantum gravity. The analysis is performed in the spirit of a mind map, which represents the architectural approach to the studied theory, being a natural way to describe the properties of a complex system. We hope that the constructed graphs (maps) will turn out to be helpful in uncovering the global picture of quantum gravity as a particular complex system and serve as a useful guide for the researchers.
\end{abstract}

Keywords Causal dynamical triangulations · Horava-Lifshitz gravity · Asymptotic safety - Loop quantum gravity - Deformations of relativistic symmetries · Phase diagram $\cdot$ Spectral dimension

Tomasz Trześniewski

tbwbt@ift.uni.wroc.pl

1 Institute of Physics, Jagiellonian University, Łojasiewicza 11, 30-348 Kraków, Poland

2 Institute for Theoretical Physics, University of Wrocław, Pl. Borna 9, 50-204 Wrocław, Poland 


\section{Introduction}

For around 100 years $^{1}$ of constantly branching research, the landscape of quantum gravity investigations has become very broad and diverse. Nowadays it contains many scattered building blocks — conceptual dilemmas and conjectures, novel mathematical tools, models at the nascent stage of development and theories that are already quite extended. The usual perspective is that we have distinct, competing approaches to the construction of quantum gravity as well as the modification of classical general relativity (assuming that the latter is a useful intermediate step), which arose partly due to the scarcity of experimental guidelines. They can be more or less sorted in several ways, e.g. some formulations of the quantum theory are classified as canonical and some as covariant, depending on the applied procedure of quantization. One possible realization of such a taxonomy of (modified) classical and quantum gravity has been depicted in Fig. 1 but it should be understood as an outline rather than a precise diagram. In the proposed scheme we roughly divide quantum gravity approaches and the more general theoretical frameworks according to whether they are built around the assumed fundamental degrees of freedom or represent some type of the effective description (at least at their current stage of development). There is also included a spectrum of potential classical approximations to the theory, consisting of general relativity and its various modifications, which are extensively reviewed in e.g. [2,3]. Certain frameworks can be seen as reductions or developments of others, sometimes due to the synergy of different branches of research, but for simplicity we show only a few links (and we do not include different versions of quantum cosmology). In general, we have restricted here to the approaches that are either most popular or especially interesting in the context of issues that will be considered in this paper. However, simple taxonomy can not help to address the problem of consistency or contradiction between various existing models.

On the other hand, as we will argue in the next sections, some sort of convergence in physical predictions of various frameworks has recently started to become apparent. If a lucky coincidence is excluded, this may indicate significant underlying connections. Moreover, there is an interesting idea to consider (see especially Sect. 2 and references therein) that (quantum) gravity is actually, or at least can be treated as, a complex system, which exhibits emergent phenomena. Its fundamental degrees of freedom might behave in a very different way than the gravitational field at some effective level. Then individual models could be potentially applied to different layers of the theory and in this sense become unified. In order to explore all of these possibilities we should employ a more architectural way of thinking, by which we mean trying to uncover the hypothetical structure of the still incomplete theory and to understand its internal functions. This contrasts with what may be called the engineering approach, in which independent groups of researchers keep working on their rival theories and which has dominated quantum gravity over the years. In our opinion, only an appropriate combination of both architecture and engineering is able to bring us to the destination point.

\footnotetext{
${ }^{1}$ The importance of quantum effects in gravity was noted by Einstein already in 1916 [1].
} 


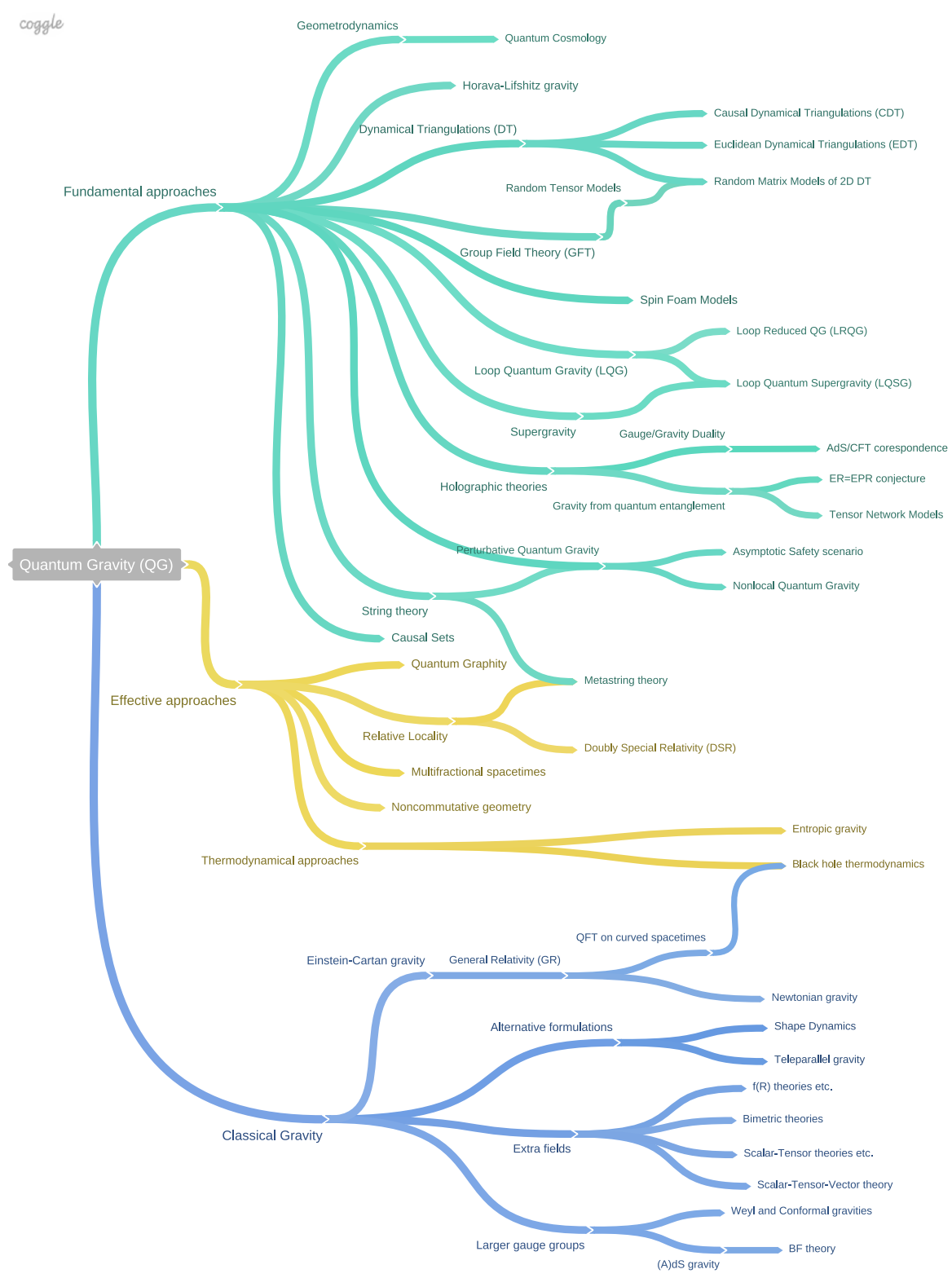

Fig. 1 A simplified taxonomy of approaches to quantum gravity

The architecture considered in this paper is not a very sophisticated one but every story has its beginning. In order to represent the structure and phenomena of the discussed theory we will apply the idea of a mind map. The nodes of such a graph (playing the role of bricks) will denote different models of quantum gravity as well as their features and known results. The links (which are like mortar) will tell us 


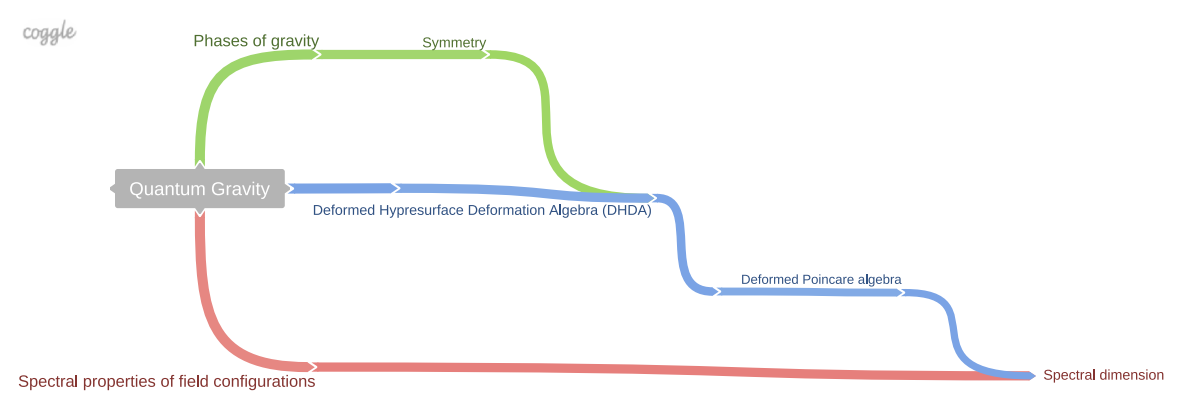

Fig. 2 Exemplary architecture of some aspects of quantum gravity

which of these elements are directly interrelated. The arrow of a link will not mean an implication but show a direction in which the graph should be read.

In Fig. 2 we present a piece of the architecture that we are going to study here. The initial node in our map is "quantum gravity". From there one can pass to the concept of "phases of gravity". Indeed, gravity is a system with a huge (perhaps infinite) number of interacting degrees of freedom. Systems of this kind, occurring in the physics of complex systems, generically exhibit such emergent phenomena as the nontrivial phase structure. Various phases can arise, depending on the surrounding environment. The particular feature distinguishing different phases is a "symmetry"-our second building block. An analogy that can be recalled here is the difference between the solid and liquid states of water. The symmetries of the liquid state are the invariance under rotations and translations, while in the solid state these symmetries are partially broken due to the formation of the crystal structure.

The notion of symmetry can be expressed in the algebraic terms by introducing the generators of symmetry transformations. For example, the generators of rotations form the $\mathfrak{s o}$ (3) algebra. This leads us to the third block of our construction, namely the "Deformed Hypersurface Deformation Algebra" (DHDA). Let us explain how does it arise. Classical theory of gravity-General Relativity - tells us that physics does not depend on the choice of a coordinate system. Such a property is a symmetry of the theory, which is known as general covariance. The generators of this symmetry (the scalar and vector constraints) form the first class algebra, the so-called Hypersurface Deformation Algebra (HDA). On the other hand, when quantum gravity effects are taken into account, the structure of HDA may become deformed, so that it is replaced by a certain DHDA.

HDA encodes the symmetry of any pseudo-Riemannian manifold but each manifold locally reduces to Minkowski spacetime, whose symmetries are described by the Poincaré algebra. The Poincaré algebra is, therefore, a sub-structure of HDA. This generalizes to the case of an arbitrary DHDA, which is expected to reduce to the corresponding deformation of the Poincaré algebra. Various versions of such a quantum deformed Poincaré algebra have been considered in the context of quantum gravity. It shows how the small scale structure of spacetime is modified by the quantum gravity effects. Another simple way in which these effects could manifest is a change in the effective dimensionality of spacetime. The latter phenomenon has been studied in 
different approaches to quantum gravity by investigating the variability (as a function of scale) of the dimension of spacetime, usually defined as the Hausdorff or spectral dimension. We will focus here on the latter, which quantifies the spectral properties of a given field configuration and explicitly depends on the form of the (deformed) Poincaré algebra and the associated Laplace operator. In the mathematical sense, the spectral dimension belongs to the "spectral properties of field configurations".

In the next sections we will gather the ingredients necessary to built a more detailed realization of the architecture introduced above, which will be presented in Sect. 8. It should be stressed that in this realization we restrict to a particular selection of approaches from Fig. 1, especially the ones that we are most familiar with. The obtained construction will be hopefully extended in the sequel to this paper, where we would like to include other aspects of quantum gravity. Here we focus on understanding the relationships between: phases of gravity, the running dimension of spacetime, deformations of relativistic symmetries and the nonlinear structure of phase space.

\section{Phases of gravity}

There is growing evidence (see e.g. [4-7]) that the gravitational field can exist in different phases (i.e. macroscopic states). This is actually not surprising, since fields as well as systems of particles usually form various phases, invariant under different sets of symmetries and determined by the initial conditions and interaction with the environment. The so-called analogue gravity models [8] have shown many interesting similarities between gravity and condensed matter physics. Furthermore, it is becoming apparent that the quantum gravitational phenomena might also be explained in terms of the quantum many-body systems, which naturally reflects their non-trivial phase properties [9]. In the case of gravity, the first indication of the nontrivial phase structure came from the numerical results of Euclidean Dynamical Triangulations (EDT), formulated within the path integral quantization approach. EDT facilitate the computations by discretizing spacetime into simplices, with the help of the Regge calculus, and making the Wick rotation to the Euclidean domain. Consequently, the quantum gravitational field can be described by a statistical ensemble and studied using the Monte Carlo simulations, which allow to find the equilibrium configurations for different values of the coupling constants. An equilibrium is equivalent to a classical path, while thermal fluctuations correspond to quantum fluctuations around it.

The $(2+1)$-dimensional EDT were the first to predict two distinct phases of gravity, separated by a first order phase transition [10]: the so-called branched polymer phase and the crumpled phase. The investigations were later extended to $3+1$ dimensions, showing that the above phase structure is preserved in this case [11]. Gravitational phases can usually be distinguished by the effective dimensionality of spacetime (which will be discussed in more detail in Sect. 4). In particular, in the crumpled phase of EDT the Hausdorff dimension behaves as $d_{H} \rightarrow \infty$, while in the branched polymer phase we have $d_{H}=2$ and the (constant) spectral dimension $d_{S}=\frac{4}{3}$. EDT in the standard formulation does not exhibit a phase with "extended" four dimensional spacetime, which would describe a semiclassical solution of the theory. It has recently 
been verified [12] that such a phase does not emerge even after introducing a nontrivial measure in the path integral.

Further information about the presumed phase structure of gravity comes from simulations of Causal Dynamical Triangulations (CDT) [13-15]. This improved approach is constructed by imposing a causal structure on configurations of EDT. The causality condition is realized owing to the introduction of a preferred foliation of spacetime, which may seem to be somewhat restrictive. However, it has also been argued [16] that the preferred foliation is not a necessary ingredient but only a convenient choice and the results of CDT are indeed sufficiently generic. One of the remarkable consequences of CDT is appearance of the "extended" four dimensional phase. In total, three main phases, called $A, B$ and $C$, have been observed. An average spacetime configuration in the phase $C$ is a well extended blob characterized by the Hausdorff dimension $d_{H} \approx 4$. Moreover, most of the vertices of such a triangulation have relatively small valence, while the maximal valence in a spatial slice grows proportionally to its volume [17]. The notion of geometry emerges naturally via a global coordinate system that can be introduced to parametrize the graph representing a given triangulation. The graph corresponding to the phase $B$ is lacking the latter property since in this case the typical valence of vertices is too large. According to the numerical results of $4 \mathrm{~d} C D T$, this phase is characterized by the Hausdorff and spectral dimensions tending to infinity, $d_{H} \rightarrow \infty, d_{S} \rightarrow \infty$. Therefore, the phase $B$ is often perceived as a counterpart of the crumpled phase in $4 \mathrm{~d}$ EDT. On the other hand, the phase $A$ shares some properties of the branched polymer phase. However, a detailed study of this phase has not been performed so far.

The phases $C$ and $A$ are separated by a first order transition line [18], while the transition between $C$ and $B$ is of the second order [19]. The order of the $A-B$ transition is unknown. The $C-A, C-B$ and $A-B$ transition lines meet at the triple point. Furthermore, recent results in $4 \mathrm{~d}$ CDT suggest that there exists an additional phase inside the phase $C$, called the bifurcation phase [17], which seems to exhibit the phenomenon of the metric signature change [20]. Further analysis is required to understand the nature of this new region on the phase diagram.

It should also be stressed here that different types of the order parameter can be applied in order to discern the phases and transitions between them. Each order parameter is sensitive to certain particular features (including symmetries) of a given field configuration. The $A, B, C$ phases in CDT are detected using the heuristically introduced "average geometry" parameter. However, in order to observe the bifurcation phase, which is a subphase of the phase $C$, a more sophisticated parameter has to be chosen. Therefore, the number of perceived distinct phases depends on what type of the order parameter is considered.

Interestingly, it has been shown [21,22] that three phases similar to those present in 4d CDT appear also in the so-called Quantum Graphity [23] model of the Planck scale physics. In this case the (extended/semiclassical) phase $C$ is realized at the minimum of energy and is the most stable configuration (i.e. a vacuum). The phases $A$ and $B$ can be reached by departing from the minimal energy. Furthermore, the transition between the high temperature non-geometric phase and low temperature geometric phase shares properties of the transition between the crumpled phase $B$ and the geometric phase $C$ in CDT. In this context the term geometrogenesis has been coined and 
possible observational relevance of such gravitational phase transitions has been studied [24]. One can say that the $B-C$ transition in CDT provides a concrete realization of the geometrogenesis discussed in the context of Quantum Graphity. A recent study [26], which also explores the relation between geometrogenesis and the ultralocal limit of gravity (we will discuss the latter in Sect. 4).

Transitions between different phases of gravity in principle may be of the first, second or higher order. Among them, the second order transitions deserve a special attention. The reason is that at such a transition the correlation function of a given order parameter diverges and the theory becomes scale invariant. Consequently, field configurations in the continuum limit (of discretization) should be described by a conformal quantum field theory. This concerns either the full spacetime geometry or only its spatial part.

Therefore, it is clear that GR, which does not satisfy the conformal invariance, cannot describe the classical state of gravitational field at a second order transition point (or line, as in the case of CDT). We may suppose that GR is just a (semi)classical theory of the extended phase of gravity, while at the phase transitions, or in other phases, the fundamental quantum theory of gravity reduces to certain effective theories that are different from GR. Let us compare this possibility with the phase structure of water. The fundamental quantum Hamiltonian of water is the same for every phase but various effective descriptions are needed in individual phases. In particular, the Clapeyron equation provides a sufficient effective model in the low-density gaseous state. On the other hand, the liquid state is well modeled as a non-compressive fluid, characterized by the Navier-Stokes equation. Both equations may be called the equations of state, as the relations between variables that are satisfied in a given phase. Analogously, perhaps the Einstein equation is an equation of state that is valid only in the extended phase of gravity. Then different equations of state would be required as the proper description of gravity in other regions of phase space.

Coming back to the second order phase transition, a choice of the proper equation of state in such a case depends on whether it is full spacetime that is conformally invariant or only its spatial slices. For the full conformal invariance one might consider e.g. the Weyl gravity. ${ }^{2}$ Therefore, a classical theory of gravity different than GR, such as the Weyl gravity (investigated so far due to the purely theoretical reasons), can potentially arise as an effective description of the gravitational field at the second order transition. On the other hand, when the conformal invariance holds only at the level of spatial hypersurfaces, the so-called Cotton tensor will be more adequate to consider. The latter situation occurs in the Hořava-Lifshitz approach to quantum gravity [26]. In this context it is worth to mention that, as analyzed in [27], the Hořava-Lifshitz theory and its precursor-the Lifshitz scalar, have the phase structure analogous to the one observed in CDT for the "average geometry" order parameter.

The existence of various phases of gravity has been also predicted within Group Field Theory (GFT) [28], loop quantum gravity and Spin Foam models. Worth mentioning here is that, although strictly related, these three approaches are characterized

\footnotetext{
2 The action of the Weyl gravity is $S=\frac{1}{16 \pi G} \int d^{4} x \sqrt{-g} C_{\mu \nu \alpha \beta} C^{\mu \nu \alpha \beta}$, where $C_{\mu \nu \alpha \beta}$ denotes the Weyl tensor. The Weyl tensor is invariant under a conformal transformation $g_{\mu \nu} \rightarrow g_{\mu \nu}^{\prime} \equiv \Omega^{2}(x) g_{\mu \nu}$.
} 
by different Hilbert spaces and dynamics. A GFT model defined on the group U $(1)^{\times 3}$ was studied with the help of the Functional Renormalization Group and found to have the nontrivial phase structure, containing the Gaussian and non-Gaussian fixed points [29]. Furthermore, the emergence of bosonic condensate phases of GFT, which are probably associated with the vacuum states different from the Fock vacuum, has been observed and interpreted in the cosmological terms, cf. [30,31] and references therein. Such GFT condensates are another potential realization of the geometrogenesis mentioned above. Meanwhile, the phase diagram of Spin Foam models was tentatively derived in [32]. An indication of distinct phases in LQG can also be noticed within investigations of its vacuum. In the recently proposed new formulation of LQG [33] there appears a new vacuum state, the so-called BF-vacuum $|0\rangle_{B F}$. Such a ground state (given by a spin network with a large number of vertices) has the constant curvature and is a sort of the condensate state, similar to the ones considered in GFT. This contrasts with the Ashtekar-Lewandowski vacuum $|0\rangle_{A L}$, for which no single node of a spin network exist. The two vacuum states correspond to two inequivalent representations of the LQG algebra of canonical variables. Therefore, they are possibly associated with different phases of the gravitational field. Finally, the Random Tensor Models, which are the higher dimensional extension of the Random Matrix Models used to study 2D quantum gravity and can also be seen as a simple version of GFT, indicate the non-trivial phase structure via their observed critical behavior (in the large $N$ expansion) $[34,35]$. Further discussion and examples of the many-body aspects of quantum gravity and the related non-trivial phase structure can be found in e.g. $[6,9,36]$.

\section{Asymptotic safety and UV fixed point}

There is much more to say about the second order phase tradition in gravity. Namely, this type of a transition is associated with existence of the critical point (or critical line). The criticality leads to the hypothesis of asymptotic safety [37,38], first proposed by Weinberg (see below).

General Relativity is perturbatively nonrenormalizable, which is caused by the dimensionful character of the Newton coupling constant. In a perturbative expansion of the probability amplitudes there occur additional UV divergences, which require a regularization by adding the appropriate counterterms to the Hamiltonian. Consequently, new coupling constants (that multiply counterterms) appear in the quantum theory and have to be fixed in order to make predictions. Since values of these couplings are a priori unknown, the theory is lacking the predictive power.

While GR is perturbatively nonrenormalizable, there is still a possibility that it is nonperturbatively renormalizable. This is precisely the idea of asymptotic safety. Namely, the conjecture put forward by Weinberg [37] is that if there exists a nonGaussian UV fixed point in the Renormalization Group (RG) flow and it can be reached by trajectories belonging to a finite dimensional critical surface, then only a finite number of couplings (equal to the dimension of the critical surface) has to be fixed to cancel the divergences of GR. Investigation of such a scenario has attracted significant attention in recent years [39].

Analysis of various effective quantum actions of GR (containing higher order derivatives) supports the asymptotic safety conjecture by indicating existence of a 
critical point (nontrivial fixed point) for the considered actions. However, in order to prove the conjecture, all allowed effective actions should be taken into account. If the conjecture is correct, then there would be no formal necessity to introduce new degrees of freedom for gravity at the Planck scale and GR could be quantized just as it is. Furthermore, in the latter case there would be no interpretation of GR as an effective theory, valid only at the energy scales below the Planck energy, as one may expect from its perturbative nonrenormalizability. Nevertheless, it is important to stress that asymptotic safety does not rule out that GR actually is an effective theory. It is still possible that more fundamental degrees of freedom have to be introduced due to the underlying physical reasons. Such a situation happens in the theory of hydrodynamics, which despite being simultaneously perturbatively nonrenormalizable and asymptotically safe, does not provide the accurate description of liquid water at the atomic scale or near the phase transitions.

Searching for the critical behavior of the gravitational field gains, therefore, an additional motivation. In fact, the CDT results discussed in the previous section seem to support the asymptotic safety conjecture. This is due to the presence of the second order transition line. Preliminary results of the RG analysis in CDT [40] indicate that the triple point, which ends the second order $C-B$ transition line, is probably related to the UV critical point via the continuum limit. However, then there may appear the anisotropic scaling between spatial and temporal directions, as suggested in [40]. In such a situation, RG trajectories would "flow" from approximately the centre of the phase $C$ towards the second order transition line and finally converge at the triple point. Further numerical simulations have to be performed in order to verify these findings.

The type of anisotropy that is needed to obtain a UV fixed point in CDT is exactly the one assumed in the Hořava-Lifshitz gravity (HLG) approach to quantum gravity. HLG is a generalization of GR whose action (in the UV) is invariant under the anisotropic scaling of spacetime coordinates [26]

$$
\mathbf{x} \rightarrow b \mathbf{x}, \quad t \rightarrow b^{z} t
$$

with an arbitrary constant $b$ and the critical exponent $z$. The standard GR can be recovered for $z=1$, while for $z=3$, at the so-called Lifshitz point of the RG flow, the theory in $3+1$ dimensions (similar to a Lifshitz scalar field theory) becomes power-counting renormalizable. This and other choices of the critical exponent will be discussed in more detail in the next section.

An extremely promising possibility is that the UV fixed point appearing in the asymptotic safety scenario, the triple point on the CDT phase diagram and the Lifshitz point $(z=3)$ of HLG are actually the same critical point. Besides the arguments presented above, further support for such a case comes from the results for the spectral dimension of spacetime.

\section{Spectral dimension and phases of gravity}

A method to characterize the structure of quantum spacetime that has been widely used in recent years are calculations of the effective number of spacetime dimensions, 
especially applying the notion of the spectral dimension. This particular definition of the dimension employs the fact that the return probability of diffusion (random walk) to the same point strongly depends on the dimensionality of a manifold on which it is considered. In particular, in Euclidean space $\mathbb{R}^{d}$ the averaged (over the whole space) return probability scales as $P(\sigma) \propto \sigma^{-d / 2}$, where $\sigma$ is the auxilliary diffusion time. By analogy with the scaling of $P(\sigma)$ in Euclidean space, the spectral dimension of a manifold is introduced as

$$
d_{S}:=-2 \frac{\partial \log P(\sigma)}{\partial \log \sigma},
$$

so that for Euclidean space we have simply $d_{S}=d$. Since the above definition concerns Riemannian manifolds, spacetime first has to be Wick-rotated. Moreover, it should be mentioned that the expression (2) does not take into account the effects of topology or curvature.

The scaling of $P(\sigma)$ is a probe of departure from the Euclidean geometry, which is associated with the spectral properties of a given manifold. In order to determine this quantity one considers a fictitious diffusion process, described by the heat equation

$$
\frac{\partial}{\partial \sigma} K(\mathbf{x}, \mathbf{y} ; \sigma)=\Delta_{x} K(\mathbf{x}, \mathbf{y} ; \sigma)
$$

where $\Delta_{x}$ denotes the appropriate Laplace operator and the initial condition $K(\mathbf{x}, \mathbf{y} ; \sigma)=\delta^{(d)}(\mathbf{x}-\mathbf{y})$ is assumed (when the manifold is flat). The heat kernel $K(\mathbf{x}, \mathbf{y} ; \sigma)$, which is the solution to (3), allows to calculate the average return probability

$$
P(\sigma):=\int d x K(\mathbf{x}, \mathbf{x} ; \sigma)=\int d \mu(p) e^{\sigma \Delta_{p}},
$$

where $\Delta_{p}$ is the momentum representation of $\Delta_{x}$ and $\mu(p)$ an invariant measure on momentum space. For quantum spacetimes it is required that at large diffusion times (probing large scales) the classical value of the dimension is recovered. On the other hand, for small times the small scale structure of spacetime is explored, corresponding to the UV limit of the theory, which may lead to the nontrivial behavior of the dimension.

Indeed, running of the spectral dimension as a function of scale has been found in such approaches to quantum gravity and models of quantum spacetime as: CDT [41,42], Hořava-Lifshitz gravity [43], asymptotic safety scenario [44], nonlocal quantum gravity [45], broadly understood loop quantum gravity [46,47] (so far, only at the kinematical level), causal sets [48,49], multifractional spacetimes [50], noncommutative spacetimes [51-53] and spacetimes characterized by a deformed hypersurface deformation algebra [54] (see the next section). In almost all of the cases the obtained results show a dimensional reduction in the UV limit, usually to $d_{S} \approx 2$, which is the value that makes gravity power-counting renormalizable. This is also in agreement with what is expected at the UV fixed point in the asymptotic safety conjecture [44]. Other results may be associated with different phases of the theory. In general, spacetimes exhibiting the phenomenon of the running dimension are called multiscale. Various relevant aspects of the dimensionality of spacetime are discussed e.g. in [55] and within a recent review of multifractional spacetimes [56]. 


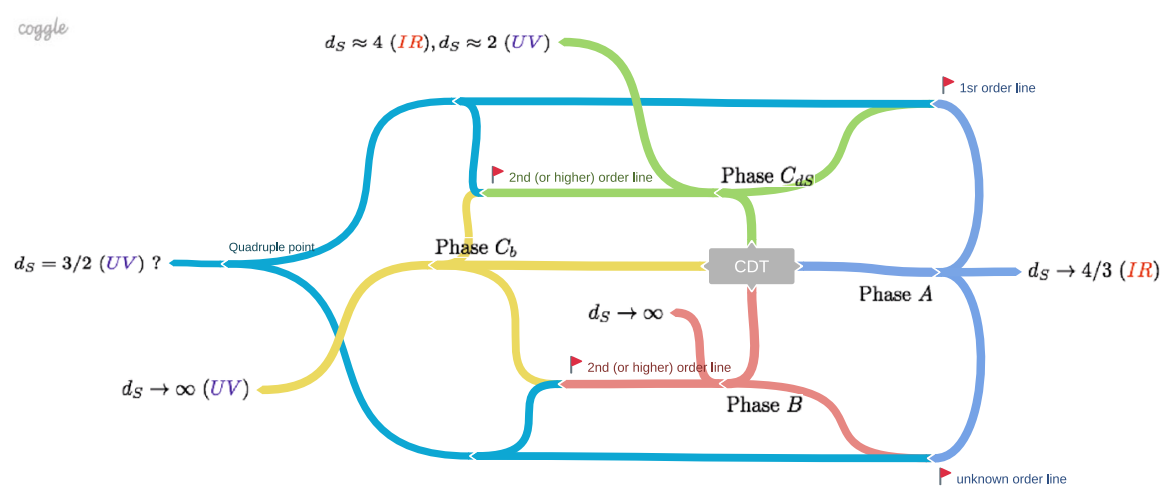

Fig. 3 Phase structure of CDT and asymptotics of the spectral dimension

In the case of CDT, each of the four phases (taking into account the bifurcation phase) is characterized by a different scale dependence of the spectral dimension. All phases and transitions between them have been collected on a graph in Fig. 3 together with the corresponding IR and UV limits of the spectral dimension.

As it was already mentioned in Sect. 2, in the non-geometric phase $B$ the UV value of the spectral dimension tends to infinity. This is a consequence of the extremely high connectivity between different points of space. It takes only a few Planck steps to go from one point to any other since for small diffusion times (for small number of steps) there is always a huge number of neighboring points. Spacetime is effectively very high dimensional, which is reflected in the spectral dimension. On the other hand, it is also easy to return in several steps to the starting point. Therefore, while the spectral dimension is sharply peaked at small diffusion times, it quickly falls to zero for larger times, as it was indicated by simulations performed in CDT. This shows that the crumpled spacetime configuration in the IR looks like a single space-time point.

Spacetime in the extended phase $C$ has a completely different structure, which can be interpreted as semiclassical. At large scales, i.e. in the IR limit, the spectral dimension saturates at $d_{S} \approx 4$, recovering (when a contribution of the assumed compact topology is subtracted) the value from the classical theory. In the seminal paper [41] it was also discovered that the dimension monotonically decreases with scale and small scales (the UV limit) are characterized by the value $d_{S} \approx 2$. Based on the requirement of consistency, one may speculate that the same behavior occurs at the triple point on the phase diagram but this presumption has to be verified by further numerical studies. Furthermore, as more extensive simulations have recently shown [42], the UV limit of the spectral dimension actually depends on a location within the phase $C$. In particular, it has been found that the value $d_{S} \approx 2$ is being measured in a region lying deep inside the phase $C$. However, if one approaches the transition line to the phase $A$, the spectral dimension decreases to $d_{S} \approx \frac{3}{2}$, which probably reflects the renormalization group flow.

Finally, the spectral dimension in the phase $A$ has not yet been a subject of systematic studies. In the numerical simulations spacetime in this phase appears to behave as a sequence of causally disconnected spatial slices. A preliminary analysis of the spectral 
dimension indicates that at large scales it becomes $d_{S} \rightarrow \frac{4}{3}$ [42], which is the same as for the branched polymer phase in EDT.

Meanwhile, in HLG (the Hořava-Lifshitz gravity) it has been found that the spectral dimension depends on the value of the (running) critical exponent $z$ and is given by [43]

$$
d_{S}=1+\frac{D}{z}
$$

where $D$ denotes the topological dimension of space, which we fix here as $D=3$ (i.e. we consider $(3+1)$-dimensional spacetime). It is clear that the classical case with $d_{S}=4$ is correctly recovered for $z \rightarrow 1$. In turn, at the Lifshitz point $z \rightarrow 3$ the spectral dimension reduces to $d_{S}=2$. The latter result coincides with the UV limit of $d_{S}(\sigma)$ in the central region of the phase $C$ in CDT. Indeed, at least in the case of $2+1$ dimensions, it has been shown to high accuracy [57] that HLG can reproduce the spectral dimension in the above region on the CDT phase diagram for the range of scales lying between the UV and IR (although this is not enough to prove that the small scale structure of spacetime in the two theories is identical [58]). Moreover, the case of $d_{S}=\frac{3}{2}$, which occurs in $3+1$ dimensions in both CDT and EDT, in the Hořava-Lifshitz gravity is obtained for $z=6$. Analysis of the theory with this value of $z$ has not been carried out so far. Furthermore, in Hořava-Lifshitz gravity the value of spectral dimension corresponding to the branched polymer case (or CDT phase A) is obtained for $z=9$.

There are two more special situations in HLG that should be explored, namely $z \rightarrow 0$ and $z \rightarrow \infty$. In particular, analysis of the scaling properties of the gravitational action leads to the conclusion that the case $z \rightarrow 0$ realizes the so-called ultralocal limit [59] of gravity (see also Sect. 5), in which spacetime splits into a congruence of causally independent worldlines. This is a consequence of suppressing the spatial derivatives in the action, so that only the kinetic and cosmological constant terms remain. According to the formula (5), the value of the spectral dimension in such a limit will tend to infinity, $d_{S} \rightarrow \infty$. The result may seem counterintuitive since in the ultralocal state of spacetime the spectral dimension is expected to reduce to $d_{S}=1$, corresponding to the remaining single direction. The possible explanation is found by considering the phase $B$ of CDT, which can be seen as a particular realization of the ultralocal state, characterized by the anisotropic scaling $z \rightarrow 0$ (see the next paragraph). As we already discussed, in the phase $B$ the dimension at small scales becomes $d_{S} \rightarrow \infty$ but at sufficiently large scales spacetime is effectively a point.

Comparing the ultralocal state and the phase $B$ of CDT one can suspect that they are closely related. Indeed, it has been shown [27] that when the phase $B$ is approached from the phase $C$, the ratio of lengths of spacelike and timelike simplicial links is decreasing. Such an effect is associated with the collapse of lightcones into worldlines, which is exactly what happens in the ultralocal limit. The ultralocality of the phase $B$ is, however, partially obscured due to the constraints that are imposed at the level of numerical simulations. The crucial assumption of CDT is that the (compact) topology of spatial slices is preserved, which is necessary to ensure that the causality in quantum spacetime is not violated. However, this constraint also prevents the 


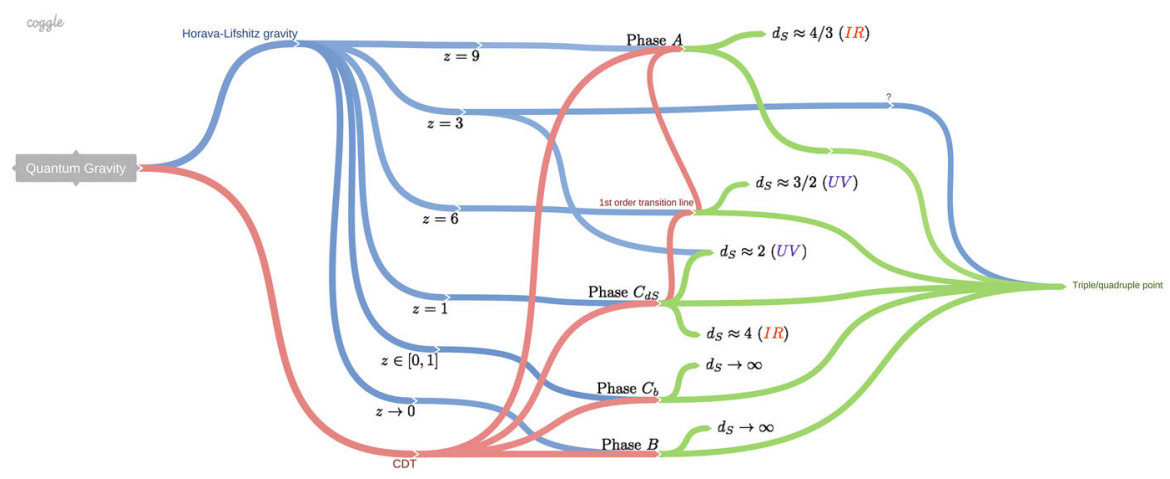

Fig. 4 Relations connecting CDT and the Hořava-Lifshitz gravity

gravitational configuration from achieving the "extended" ultralocal state, in which all points of space evolve as disconnected universes. Another restriction, introduced for computational reasons, is that the total number of simplices (or the average of it) is fixed, being controlled by the cosmological constant, which plays the role of a chemical potential in the statistical ensemble. Due to the combined effect of the above constraints in CDT, an attempted simulation of the ultralocal state leads to a pointlike universe pierced by a timelike straw (the latter may or may not have the physical meaning). The dimensionality of space in the IR is correctly zero, which results from the crumpled structure in the UV. The only possible real difference with respect to the ultralocal state in HLG is the behavior of the time direction. Depending on whether the straw observed in numerical simulations is interpreted as a physical feature or a numerical artifact, the time direction stretches a single dimension or disappears, respectively. Let us also mention that since the phase $B$ corresponds to the anisotropic scaling $z=0$, while the phase $C_{d S}$ to $z=1$, we conjecture that the (sub-)phase $C_{b}$ should correspond to the scaling that interpolates between these two values of $z$. However, the properties of the phase $C_{b}$ have not yet been completely studied.

Coming back to the Hořava-Lifshitz gravity, the last case to consider is the critical exponent $z \rightarrow \infty$, for which the spectral dimension $d_{S} \rightarrow 1$. While the dimension behaves here as it can be expected for the ultralocal state, field configurations are not characterized by vanishing of the spatial derivatives. It is quite the opposite, since the action invariant under the $z \rightarrow \infty$ scaling contains derivatives of the infinitely high order, possibly describing an extremely strongly correlated configuration. Physical properties of the case $z \rightarrow \infty$ deserve a separate detailed analysis, which still has to be carried out.

Concluding the discussion, we can tentatively consider that the Hořava-Lifshitz gravity and Causal Dynamical Triangulations are connected by the network of relations depicted in Fig. 4. In this section we actually restricted mainly to the above two approaches to quantum gravity. An outline of the more inclusive map of quantum gravity that is based on predictions for the dimensionality of spacetime (using also other notions besides the spectral dimension) has recently been proposed in [60]. 


\section{Deformations of the hypersurface deformation algebra}

The symmetry of General Relativity is general covariance. In the Hamiltonian framework it is embedded in the structure of the algebra of constraints, the so-called Hypersurface Deformation Algebra (HDA). This algebra (more precisely, it is an algebroid [61]) is of the first class and therefore the constraints are given the interpretation of symmetry generators. In the case of classical GR transformations generated by the constraints coincide with the Lie derivatives. However, the evidence gathered in recent years suggests that this property may be spoiled when the Planck scale physics is taken into account.

Namely, the effects of quantum gravity can lead to certain deformations (i.e. modifications of the brackets) of the hypersurface deformation algebra. The ensuing Deformed Hypersurface Deformation Algebra (DHDA) remains of the first class and the constraints still act as the generators of gauge transformations. Nevertheless, apart from certain limits, the transformations will differ from the Lie derivatives. Consequently, the spacetime metric will no longer be a covariant object, which suggests that it does not play such a significant role here as in the classical theory. The deformations of this type have been derived through an analysis of the effective algebra of quantum constraints in loop quantum gravity. For both spherically symmetric configurations and cosmological perturbations the obtained algebra has the brackets $[62,63]$

$$
\begin{aligned}
\left\{D\left[N_{1}^{a}\right], D\left[N_{2}^{a}\right]\right\} & =D\left[N_{1}^{b} \partial_{b} N_{2}^{a}-N_{2}^{b} \partial_{b} N_{1}^{a}\right], \\
\left\{S^{Q}[N], D\left[N^{a}\right]\right\} & =-S^{Q}\left[N^{b} \partial_{b} N\right], \\
\left\{S^{Q}\left[N_{1}\right], S^{Q}\left[N_{2}\right]\right\} & =D\left[s \Omega g^{a b}\left(N_{1} \partial_{b} N_{2}-N_{2} \partial_{b} N_{1}\right)\right],
\end{aligned}
$$

where $\Omega$ is the deformation factor (affecting only the last bracket), $g^{a b}$ denotes the spatial metric and $s$ the spacetime metric signature, which is $s=1$ in the Lorentzian case and $s=-1$ in the Euclidean one. The superscript $Q$ means that the scalar constraints $S^{Q}[N]$ are themselves quantum deformed (with respect to their classical counterparts). In the case of cosmological perturbations it has been found that the deformation factor takes the following form [64]

$$
\Omega=\cos (2 \gamma \bar{\mu} \bar{k})=1-2 \frac{\rho}{\rho_{c}} \in[-1,1],
$$

with the critical energy density $\rho_{c}=3 /(8 \pi G \gamma \Delta) \sim \rho_{\mathrm{Pl}}$, depending on the Immirzi parameter $\gamma$ and the minimal surface area $\Delta$. Similarly, for the spherically symmetric configurations we have $\Omega=\cos \left(2 \delta K_{\varphi}\right)$. In both cases the expression for $\Omega$ is a cosine function of the extrinsic curvature. Such an effect originates from the so-called holonomy corrections, which are the result of replacing the Ashtekar connection by the corresponding holonomies ${ }^{3}$. However, the inverse volume corrections are to be

\footnotetext{
${ }^{3}$ The holonomy of the Ashtekar connection along a contour $\mathcal{C}$ is given by the path-ordered exponential $h_{\mathcal{C}}:=\mathcal{P}_{e} \int_{\mathcal{C}}{ }^{A}$.
} 


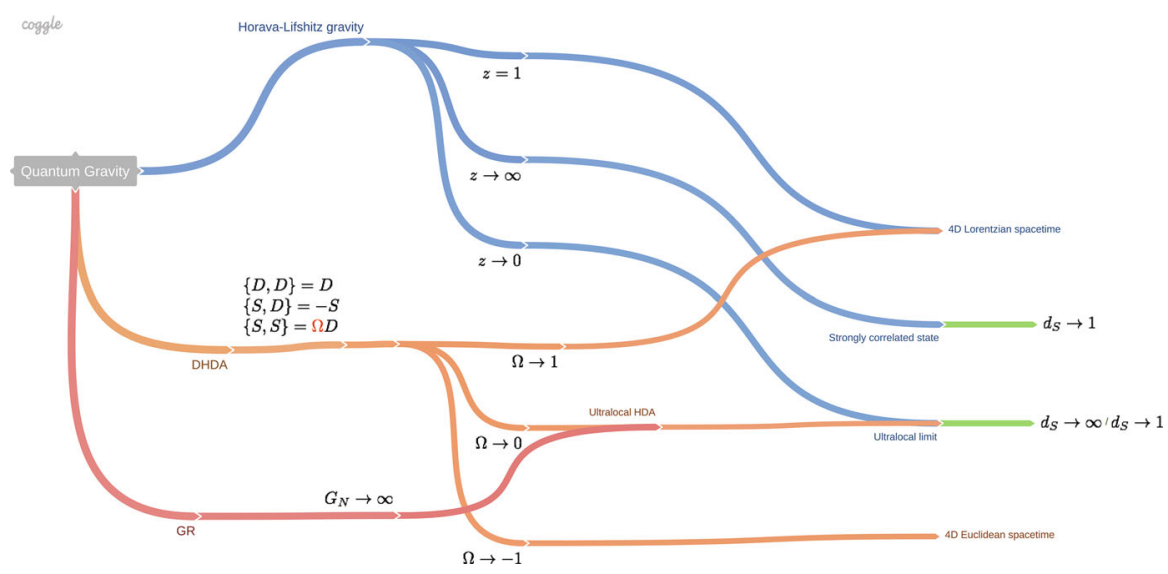

Fig. 5 Deformed HDA in the context of quantum gravity

expected as well, which will modify the expression (7) [65]. It has also been shown [66] that substituting a function $f(p)$ in the place of the kinetic term $p^{2}$ in the field Hamiltonian leads to the DHDA with the deformation factor

$$
\Omega=\frac{1}{2} \frac{d^{2} f(p)}{d p^{2}} .
$$

In particular, when the polymer quantization is applied to the canonical momentum via the function

$$
f(p)=\frac{\sin ^{2}(\lambda p)}{\lambda^{2}}
$$

(as it is done in loop quantum cosmology), one obtains $\Omega=\cos (2 \lambda p)$. $\lambda$ is here the scale of polymerization, introduced in such a way that the classical symmetries are recovered for $\lambda \rightarrow 0$. This explains the origin of the cosine form of deformations observed in the above mentioned effective models of LQG.

The graph presented in Fig. 5 contains different cases of the DHDA (6) and their relations with some other aspects of quantum gravity. Depending on the value of $\Omega$, several physical scenarios are realized. In particular, for $\Omega \rightarrow 1$ we recover classical GR with the Lorentzian metric signature. For $\Omega \rightarrow-1$ classical GR is recovered as well but with the Euclidean signature, while the case of $\Omega \rightarrow 0$ is equivalent to the ultralocal limit, discussed in the previous section. It is worth to add here that the ultralocal state can also be obtained in classical GR, by taking the strong coupling limit $G \rightarrow \infty$ [59]. Furthermore, in the sense of the BKL conjecture [67,68], the ultralocality is a general prediction when evolution of the gravitational field towards a singularity is considered. In this context it is associated with the concept of asymptotic silence [69].

As can be seen from (7), the type of DHDA obtained in loop quantum cosmology (LQC) leads to the effect of the metric signature change. When energy density of the matter content of universe reaches the value $\rho=\rho_{c} / 2$, then $\Omega$ changes its sign from positive to negative, which has the interpretation of going from the Lorentzian 
to Euclidean signature. This curious phenomenon has been a subject of several investigations in recent years [70,71]. Furthermore, the ultralocal state that emerges at $\rho=\rho_{c} / 2$ has been studied in [72]. An essential feature of the $\Omega$-deformation in LQC, pointed out in [61], is that it is actually associated with the non-Riemannian geometry of spacetime. Further understanding on this small scale structure in the regime where deformations are significant may be achieved through an analysis of the corresponding deformations of the Poincaré algebra. This issue is the topic of the next section.

\section{Connection between DHDA and the deformed Poincaré algebra}

The hypersurface deformation algebra (HDA) describes symmetries of an arbitrary pseudo-Riemannian manifold and the simplest case of such a manifold is naturally the Minkowski spacetime. The isometries of Minkowski spacetime form the Poincaré algebra, which can also be obtained in the limit of linear hypersurface deformations in the corresponding HDA. Accordingly, any modification of the standard HDA is expected to affect the structure of the Poincaré algebra. In particular, this is predicted to be the case in LQG, where the effective algebra of constraints (which is equivalent to HDA) is deformed, as we have already discussed.

It has been argued that the deformed Poincaré algebra corresponding to the DHDA (6) has the following form [54]:

$$
\begin{aligned}
\left\{J_{a}, J_{b}\right\} & =\epsilon_{a b c} J^{c}, \\
\left\{J_{a}, K_{b}\right\} & =\epsilon_{a b c} K^{c}, \\
\left\{K_{a}, K_{b}\right\} & =-s_{e f f} \epsilon_{a b c} J^{c}, \\
\left\{J_{a}, P_{b}\right\} & =\epsilon_{a b c} P^{c}, \\
\left\{J_{a}, P_{0}\right\} & =0, \\
\left\{K_{a}, P_{b}\right\} & =\delta_{a b} P_{0}, \\
\left\{K_{a}, P_{0}\right\} & =s_{e f f} P_{a}, \\
\left\{P_{a}, P_{b}\right\} & =0, \\
\left\{P_{a}, P_{0}\right\} & =0,
\end{aligned}
$$

with the deformed signature factor $s_{\text {eff }} \equiv s \tilde{\Omega}$, which is a function of the algebra generators and becomes the metric signature $s_{\text {eff }}=s$ in the limits $\tilde{\Omega} \rightarrow \pm 1$. So far, the form of $\tilde{\Omega}$ has not been determined in general but some attempts have been made. In particular, by requiring the algebra to satisfy the Jacobi identities and $\tilde{\Omega}$ to be a separable function of $P_{0}^{2}$ and $P_{i}^{2}$ (which is a convenient Ansatz), one obtains the following expression $[54,73]$ :

$$
\tilde{\Omega}=\frac{P_{0}^{2}-\alpha}{P_{i}^{2}-\alpha},
$$

where $\alpha \in \mathbb{R}$ is a free parameter associated with the energy scale of the deformation. In such a case the Euclidean mass Casimir element (with the proper classical limit) can be constructed as 


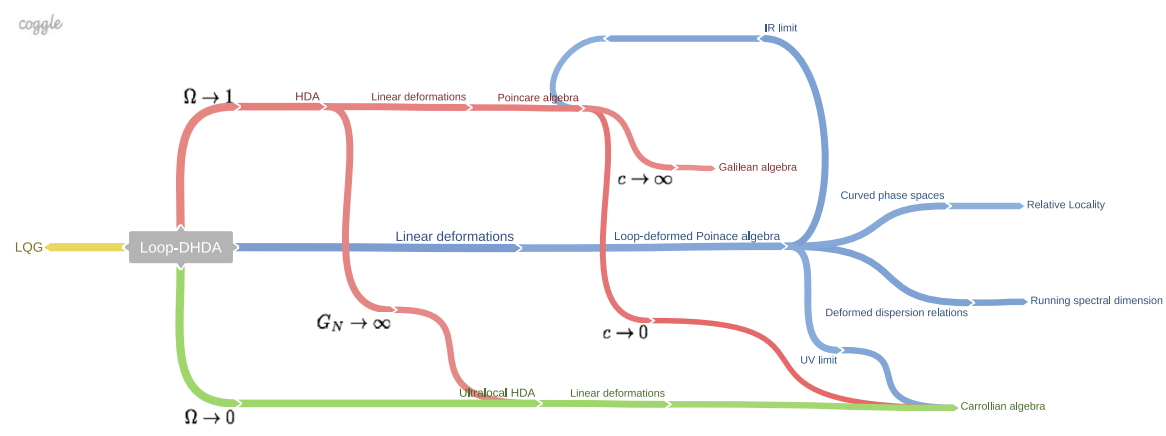

Fig. 6 From the DHDA to the deformed Poincaré algebra

$$
\mathcal{C}^{E}=\frac{P_{0}^{2}+\mathbf{P}^{2}}{1-\alpha^{-1} \mathbf{P}^{2}}
$$

and the simplest possible guess concerning the form of the d'Alambert operator on momentum space is $\Delta_{p}:=-\mathcal{C}^{E}$. Substituting the latter into (4), we are able to calculate the spectral dimension of spacetime equipped with the deformed symmetry algebra (10-18). It has been found [54] that the dimension reduces from $d_{S}=4$ at large scales to $d_{S}=1$ at short scales, reflecting the ultralocality arising in the limit $\tilde{\Omega} \rightarrow 0$.

The connection between HDA and the Poincaré algebra is a crucial element in the construction of our map of quantum gravity. When generalized to the deformed case, it allows to relate (see the end of this section) the two classes of, previously separated, approaches to the Planck scale physics: LQG and models of spacetime with deformed relativistic symmetries. This is illustrated in Fig. 6.

In particular, let us focus on the DHDA given by (6). In the $\Omega \rightarrow 0$ limit it becomes an ultralocal algebra. By the restriction to the case of linear hypersurface deformations, this algebra reduces to the so-called Carroll algebra. So far, limited amount of research has been directed toward this structure. However, as we saw, it may actually reflect the quantum gravitational effects. The Carroll algebra has actually been introduced at the classical level, where it is obtained by taking the limit of vanishing speed of light, $c \rightarrow 0$, of the Poincaré algebra [74]. This qualitatively overlaps with the observation made for DHDA, where the effective speed of propagation is found to be $v_{\text {eff }}=\sqrt{\Omega}$ and vanish for $\Omega \rightarrow 0$ [72].

The general form of DHDA defined by the brackets (6) may lead to various deformations of the Poincaré algebra. In particular, in [75] it was argued that the DHDA that was previously discussed in [63] possibly corresponds to the famous $\kappa$-Poincare algebra $[76,77]$. The latter algebra constitutes the archetypal example of quantum deformations of spacetime symmetries, which naturally appear in the semiclassical frameworks known as Doubly Special Relativity [78] and Relative Locality [79] (see below). Moreover, the form of deformations of both the Poincaré algebra and the corresponding HDA has been derived for certain multifractional spacetimes [80]. The results in these cases turn out to differ from (6). Meanwhile, the reconstruction of the appropriate DHDA in Relative Locality is an open task. An important role will 
probably be played there by the nonlinear momentum space, or nonlinear phase space, which is associated with the deformed relativistic symmetries.

\section{Nonlinear structure of phase space}

Deformations of relativistic symmetries discussed in the previous section are closely related to another concept appearing in the context of quantum gravity: momentum space or, more generally, phase space with the nontrivial geometry or topology. The origins of this idea go back to M. Born, who argued that momentum space and the space of particle positions, i.e. spacetime, in quantum physics are connected by the reciprocity symmetry. Therefore, in the regime of full quantum gravity, curved spacetime should be complemented by momentum space with similarly nontrivial Riemannian geometry [81]. Later it was observed [82] that non-vanishing curvature of momentum space leads to the noncommutativity of spacetime coordinates. This intuition was subsequently confirmed in the mathematical formalism of quantum groups, i.e. nontrivial Hopf algebras, which give a natural description of the deformed relativistic symmetries [83]. The most interesting case of such deformed symmetries is the $\kappa$-Poincaré (Hopf) algebra [76,77]. This algebra acts covariantly on the noncommutative $\kappa$-Minkowski space [84], while the momentum space corresponding to the latter is the AN(3) Lie group, which as a manifold is equivalent to half of de Sitter space $[85,86]$.

The above mathematical structures have been utilized [78,87-89] in the framework known as Doubly (or deformed) Special Relativity, which is the family of models that attempt to represent the semiclassical regime of quantum gravity characterized by the existence of two invariant scales, given by the speed of light and Planck mass. These two scales determine the geometry of flat spacetime and curved momentum space. Doubly special relativity has recently been recast and generalized into the so-called Relative Locality approach [79,90], whose underlying principle is that (at sufficiently high energies) only the full phase space is an absolute physical entity, while its decomposition into spacetime and momentum space depends on the choice of an observer. More specifically, the structure of spacetime is inferred from the dynamics of particles in (curved) momentum space. This leads to the relativity of locality of events in spacetime, i.e. physical events which have the same spacetime coordinates in the frame of a certain observer are not coincidental according to observers that are distant from the former. If we want to extend the above concept to the generally covariant case, both spacetime and momentum space should be allowed to have the nontrivial geometry. In order to study such a scenario there have been attempts to construct the action principle [91] or the Hamilton geometry [92] for particles having the nontrivial phase space. On the more fundamental level, there have been proposed [93,94] a unified description of the (dynamical) phase space geometry, which involves the symplectic form, the generalized metric (combining the metrics on spacetime and momentum space) and the locality metric (which encodes the pairing between spacetime and momentum space), satisfying the appropriate compatibility conditions. Using such a formulation the relative locality approach can also be introduced in the framework of string theory, where it is realized as a generalization to the meta-string theory. 
On the other hand, the nontrivial geometry of phase space has also been considered from the perspective of the candidate full quantum gravity theories (and not just models of quantum spacetime). For example, curved momentum space can in principle arise within the Group Field Theory approach. At the elementary level of this framework, spacetime is replaced by a quantum field defined on the background of several copies of a certain Lie group (associated with relativistic symmetries), which is also a curved manifold and whose tangent space is given by the related Lie algebra. A particle excitation of the field corresponds to a single quantum of discrete space(time), while different quanta become connected via the combinatorially nonlocal field interactions. Continuous spacetime is expected to emerge only in the semiclassical limit and there a group field theory should reduce to a certain effective field theory (describing perturbations around the classical solution) [95]. It is reasonable to suppose that the latter field will perceive some effective Lie group as configuration space and the (dual) Lie algebra as momentum space. However, one may also envisage the opposite situation and then, at the mathematical level, the difference between a given group field theory and the theory covariant under the action of the appropriate Hopf algebra will be the combinatorial structure of the former. Indeed, it has been tentatively shown [96] that a scalar field theory on $\kappa$-Minkowski space is equivalent to the effective, semiclassical approximation of a Poincaré group field theory. Another avenue that potentially leads to nontrivial phase space is the already mentioned polymer quantization scheme, commonly applied within LQG, which can result in the circular (periodic) momentum space of a quantum mechanical system [97].

One might also wonder whether the concept of nontrivial phase space should be extended to the domain of field theory, so that it is the phase space of values of a given field that has some nonlinear structure (rather than just the phase space on which a field is defined). In [98] (see also [99-101]) the authors of this paper proposed the conjecture that ordinary field theories are actually the low energy limit of theories whose phase spaces of field values are not affine spaces but manifolds with nontrivial geometry or topology. This has been called the Nonlinear Field Space Theory (NFST). As a prototypical example of such a framework we considered a scalar field theory whose phase space at every point of spacetime, or for every Fourier mode, has the symplectic geometry of a sphere (while the background spacetime is assumed to be Minkowski space). Performing quantization of the field we then obtain a number of predictions typical to the quantum gravity models. Moreover, if the assumed phase space is compact, as it is the case for a sphere, the so-called principle of finiteness [102] is automatically imposed on a given field, which automatically resolves the ubiquitous problem of UV divergences. In [103] we also preliminarily investigated the application of such a scalar field as the matter content of universe in the standard cosmological model, which turned out to lead to different interesting results.

Worth discussing is a further extension of the nonlinear phase space framework to the area of quantum gravity. In this context let us take a look at the case of LQG. The starting point of the latter approach is the formalism of Ashtekar variables, in which the connection $A$ and densitized triad $E$ are the canonical fields, whose values belong to the $\mathfrak{s} u(2)$ algebra [104]. The phase space of classical GR parametrized in terms of the Ashtekar variables is still affine. However, passing to LQG, $A$ is subject to the exponentiation and forms a holonomy, being an element of a compact group SU(2) 
[105]. Meanwhile, the fluxes constructed with the use of $E$ remain elements of the $\mathfrak{s} u(2)$ algebra, which is isomorphic to $\mathbb{R}^{3}$. Therefore, similarly as we observed above in the context of Group Field Theory, one can say that the phase space of LQG is partially curved.

On the other hand, in Sect. 2 it was mentioned that the opposite setup for holonomies and fluxes is considered in the recently proposed alternative formulation of LQG [106]. Namely, $E$ is then subject to the exponentiation, while fluxes of $A$ remain elements of $\mathfrak{s} u(2)$. The natural next step one could consider is to exponentiate both $E$ and $A$, so that the phase space per link of a spin network becomes $\Gamma=\mathrm{SU}(2) \times \mathrm{SU}(2)$, which is a compact manifold. The idea of generalizing LQG to such a case was discussed in [107]. While, so far, it has been mostly analyzed only in the $(2+1)$-dimensional theory, significant progress towards the extension of this framework to $3+1$ dimensions has recently been achieved [108-110].

\section{Synthesis: the first map}

In this section we make the first attempt to collect the results discussed above into a single map, representing relations between different approaches to quantum gravity. The map in Fig. 7 has been constructed using the architectural structure exemplified in Fig. 2. Its core elements are:

- Quantum deformations of HDA and the corresponding deformations of the Poincaré algebra. The bridge between them allows to relate models of quantum spacetime with the candidate theories of quantum gravity.

- Various phases of the gravitational field, invariant under different symmetries, which are described by the (deformed) symmetry algebras.

- The phase transition lines, meeting at the triple/quadruple point. This point is possibly related to a UV fixed point of the RG flow, which is also supposed to coincide with the Lifshitz point of HLG. The asymptotic safety conjecture is expected to be realized there.

- Nonlinear phase spaces of particles and fields, which can lead to deformations of the relativistic symmetries.

- The ultralocal state, predicted to occur in such approaches to quantum gravity as the effective LQG, CDT and Hořava-Lifshitz gravity.

\section{Summary and discussion}

In this paper, in the spirit of the architectural approach to a physical theory, we carried out our first attempt to draw a mind map of quantum gravity. It is based on the most up to date results, obtained within various approaches to the Planck scale physics.

As several researchers have observed (see Sect. 2), there are strong reasons to suppose that quantum gravity belongs to the realm of complex systems. A crucial indication is that the gravitational field is a system with a huge (perhaps infinite) number of degrees of freedom, which are subject to strongly non-linear interactions. Systems of this kind exhibit various emergent phenomena, such as the nontrivial 
Fig. 7 Map of relations between some aspects of quantum gravity, constructed on the basis of the architecture presented in Fig. 2

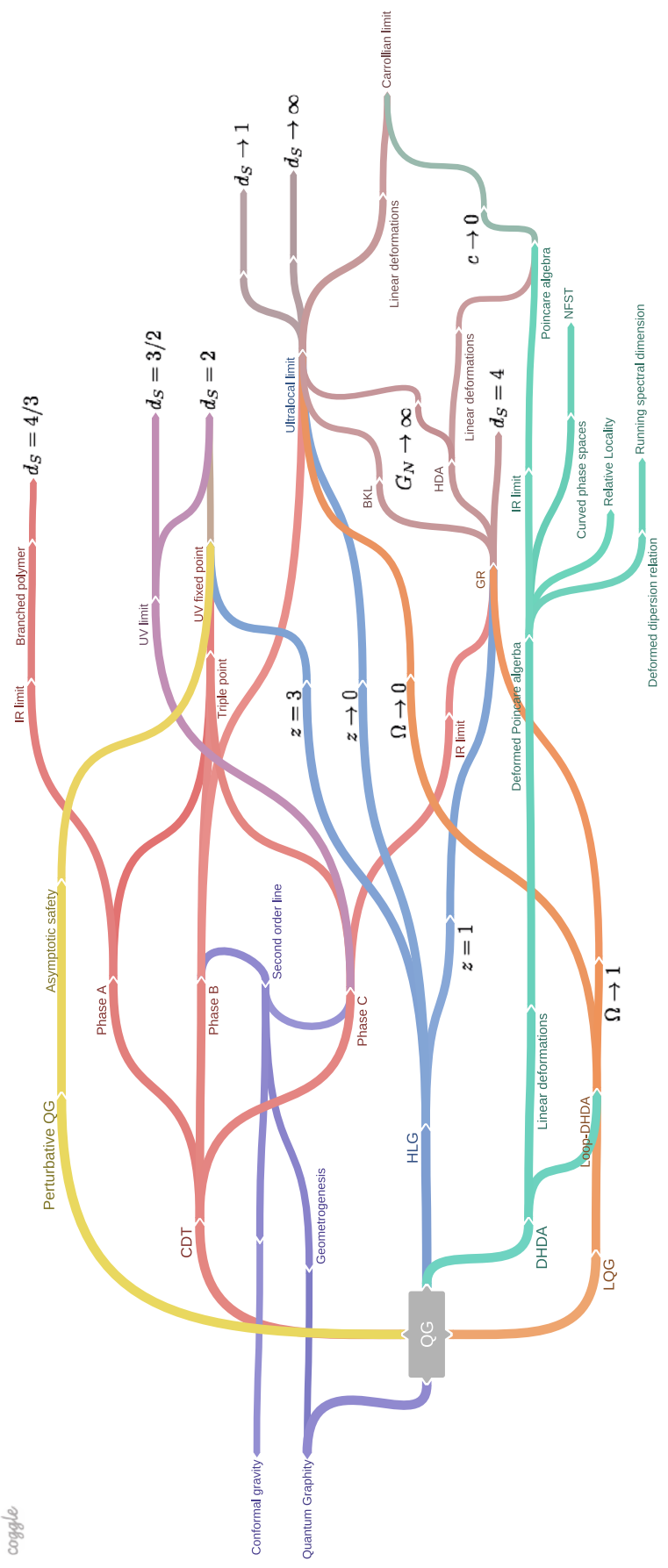


phase structure, containing phase transitions. Consequently, presumably there is no single effective description of states of quantum gravity but rather several "equations of state", corresponding to different phases. Nonlinearity implies the existence of various underlying mechanisms, each deserving a separate analysis and explanation with the help of adequate variables and a set of concepts. Similarly, the functioning of a human brain cannot be described using a single equation. Rather than that, a huge network of biochemical processes, composed of numerous contributing reactions, has to be considered.

The concept of a network, or graph, occurs naturally in the description of complex systems. It consists of two essential levels: structural and functional. The first one means that a graph serves as a method for capturing relations between elementary constituents of the complex system (e.g. connections between neurons in a brain) in the visual language. Meanwhile, at the second level a graph is a way of representing different operating processes and emergent phenomena in the system (e.g. epileptic seizures happening in a brain).

While the first (structural) point of view is extensively used in the investigations of quantum theory of gravity (e.g. spin networks, Regge calculus, tensor networks), the second one (functional) has had only a residual appearance in the quantum gravity research so far. This paper constitutes the first serious attempt to introduce the functional network approach into the domain of quantum gravity. In our discussion we have considered a simple architecture, combining such issues as the gravitational phase structure, running dimension of spacetime, asymptotic safety of the theory as well as deformations of relativistic symmetries and nonlinear phase space structures. The obtained construction indicates certain missing points, which lead to a number of open questions listed in the Appendix.

Among the most important observations that contributed to the structure of the presented map we should mention:

- Different phases of quantum gravity will have different effective descriptions (equations of state).

- There may be a direct connection between the UV fixed point in the asymptotic safety scenario, the Lifshitz point in the Hořava-Lifshitz gravity and triple (quadruple) point on the CDT phase diagram.

- A variety of approaches to quantum gravity predict the dimensional reduction of spacetime at small scales.

- A given deformed hypersurface deformation algebra in the linear limit leads to the corresponding deformation of the Poincaré algebra.

- The nonlinear structure of phase space is associated with deformations of general and special relativistic symmetries.

- The ultralocal (silent) state probably plays an important role in quantum gravity.

Quantum gravity is a fascinating jigsaw puzzle. Many of its elements are definitely still missing. The only way to find out which elements we need to look for is to put together the pieces that are already available. This has been the most important motivation behind our proposal, which deserves a further systematic extension, including additional models and aspects of quantum gravity. 
Acknowledgements This work is supported by the Iuventus Plus Grant No. 0302/IP3/2015/73 from the Polish Ministry of Science and Higher Education. TT was additionally supported by the National Science Centre Poland, Project 2014/13/B/ST2/04043.

Open Access This article is distributed under the terms of the Creative Commons Attribution 4.0 International License (http://creativecommons.org/licenses/by/4.0/), which permits unrestricted use, distribution, and reproduction in any medium, provided you give appropriate credit to the original author(s) and the source, provide a link to the Creative Commons license, and indicate if changes were made.

\section{Appendix-Open problems}

Here we collect the main open problems that emerged in the course of our investigations:

(1.) What is the behavior of the spectral dimension at the triple point on the phase diagram of CDT?

(2.) In the continuum, is the CDT triple point a Lifshitz point with $z=3$ ?

(3.) Is this point also a nontrivial UV fixed point?

(4.) What is the order of the $A-B$ phase transition in CDT?

(5.) Is the BKL conjecture realized in the phase $B$ of CDT?

(6.) What is the nature of the Horava-Lifshitz theory with the critical exponent $z \rightarrow \infty$ ?

(7.) What are the properties of the Hořava-Lifshitz theory with $z=6$, which leads to $d_{S}=\frac{3}{2}$ ?

(8.) What are the properties of the Hořava-Lifshitz theory with $z=9$, which leads to $d_{S}=\frac{4}{3}$ ?

(9.) What is the (class of) DHDA corresponding to the $\kappa$-Poincaré algebra?

(10.) Is the loop-deformed Poincaré algebra associated with certain nonlinear structure of phase space?

Finding answers to the above questions will be a significant guidance in the further extension of the map of quantum gravity.

\section{References}

1. Rovelli, C.: arXiv:gr-qc/0006061

2. Blagojević, M., Hehl, F.W.: Imperial College Press, London (2012). [arXiv:1210.3775 [gr-qc]]

3. Clifton, T., Ferreira, P.G., Padilla, A., Skordis, C.: Phys. Rep. 513, 1 (2012). [arXiv:1106.2476 [astroph.CO]]

4. Smolin, L.: Lect. Notes Phys. 461, 184 (1996). [arXiv:gr-qc/9505022]

5. Stephens, G.J., Hu, B.L.: Int. J. Theor. Phys. 40, 2183 (2001). [arXiv:gr-qc/0102052]

6. Bojowald, M.: Rep. Prog. Phys. 78, 023901 (2015). [arXiv:1501.04899 [gr-qc]]

7. Mielczarek, J.: Adv. High Energy Phys. 2017, 4015145 (2017). [arXiv:1404.0228 [gr-qc]]

8. Barceló, C., Liberati, S., Visser, M.: Living Rev. Relativ. 8, 12 (2005). [arXiv:gr-qc/0505065]

9. Oriti, D.: In: Angilella, G.G.N., Amovilli, C. (eds.) Many-body Approaches at Different Scales: A Tribute to Norman H. March on the Occasion of his 90th Birthday. Springer, New York (2018). [arXiv:1710.02807 [gr-qc]]

10. Ambjørn, J., Boulatov, D.V., Krzywicki, A., Varsted, S.: Phys. Lett. B 276, 432 (1992)

11. Białas, P., Burda, Z., Krzywicki, A., Petersson, B.: Nucl. Phys. B 472, 293 (1996). [arXiv:hep-lat/9601024]

12. Coumbe, D., Laiho, J.: JHEP 1504, 028 (2015). [arXiv:1401.3299 [hep-th]] 
13. Ambjørn, J., Jurkiewicz, J., Loll, R.: Phys. Rev. Lett. 93, 131301 (2004). [arXiv:hep-th/0505154]

14. Ambjørn, J., Görlich, A., Jurkiewicz, J., Loll, R.: Phys. Rep. 519, 127 (2012). [arXiv:1203.3591 [hep-th]]

15. Ambjørn, J., Gizbert-Studnicki, J., Görlich, A., Jurkiewicz, J., Németh, D.:arXiv:1802.10434 [hep-th]

16. Jordan, S., Loll, R.: Phys. Lett. B 724, 155 (2013). [arXiv:1305.4582 [hep-th]]

17. Ambjørn, J., Coumbe, D.N., Gizbert-Studnicki, J., Görlich, A., Jurkiewicz, J.: Phys. Rev. D 95, 124029 (2017). [arXiv:1704.04373 [hep-lat]]

18. Ambjørn, J., Jordan, S., Jurkiewicz, J., Loll, R.: Phys. Rev. D 85, 124044 (2012). [arXiv:1205.1229 [hep-th]]

19. Ambjørn, J., Jordan, S., Jurkiewicz, J., Loll, R.: Phys. Rev. Lett. 107, 211303 (2011). [arXiv:1108.3932 [hep-th]]

20. Ambjørn, J., Coumbe, D.N., Gizbert-Studnicki, J., Jurkiewicz, J.: JHEP 1508, 033 (2015). [arXiv:1503.08580 [hep-th]]

21. Wilkinson, S.A., Greentree, A.D.: Phys. Rev. D 90, 124003 (2014). [arXiv:1409.2557 [gr-qc]]

22. Wilkinson, S.A., Greentree, A.D.: Phys. Rev. D 92, 084007 (2015). [arXiv:1506.07588 [gr-qc]]

23. Konopka, T., Markopoulou, F., Severini, S.: Phys. Rev. D 77, 104029 (2008). [arXiv:0801.0861 [hep-th]]

24. Magueijo, J., Smolin, L., Contaldi, C.R.: Class. Quantum Gravity 24, 3691 (2007). [arXiv:astro-ph/0611695]

25. Mandrysz, M., Mielczarek, M.: [arXiv:1804.10793 [gr-qc]]

26. Hořava, P.: Phys. Rev. D 79, 084008 (2009). [arXiv:0901.3775 [hep-th]]

27. Ambjørn, J., Görlich, A., Jordan, S., Jurkiewicz, J., Loll, R.: Phys. Lett. B 690, 413 (2010). [arXiv:1002.3298 [hep-th]]

28. Oriti, D.: In: Ashtekar, A., Pullin, J. (eds.) Loop Quantum Gravity: The First 30 Years. World Scientific, Singapore (2017). [arXiv:1408.7112 [gr-qc]]

29. Benedetti, D., Ben Geloun, J., Oriti, D.: JHEP 1503, 084 (2015). [arXiv:1411.3180 [hep-th]]

30. Gielen, S., Sindoni, L.: SIGMA 12, 082 (2016). [arXiv:1602.08104 [gr-qc]]

31. Oriti, D.: Comptes Rendus Physique 18, 235 (2017). [arXiv:1612.09521 [gr-qc]]

32. Delcamp, C., Dittrich, B.: Class. Quantum Gravity 34, 225006 (2017). arXiv:1612.04506 [gr-qc]

33. Dittrich, B., Geiller, M.: Class. Quantum Gravity 32, 112001 (2015). [arXiv:1401.6441 [gr-qc]]

34. Bonzom, V., Gurau, R., Riello, A., Rivasseau, V.: Nucl. Phys. B 853, 174 (2011). [arXiv:1105.3122 [hep-th]]

35. Bonzom, V., Gurau, R., Ryan, J.P., Tanasa, A.: JHEP 1409, 051 (2014). [arXiv:1404.7517 [hep-th]]

36. Oriti, D.: arXiv:1803.02577 [physics.hist-ph]

37. Weinberg, S.: In: Zichichi, A. (ed.) Understanding the Fundamental Constituents of Matter. The Subnuclear Series 14, 1-52 (1978)

38. Percacci, R.: In: Oriti, D. (ed.) Approaches to quantum gravity: Towards a New Understanding of Space, Time and Matter, pp. 111-128, Cambridge (2009). [arXiv:0709.3851 [hep-th]]

39. Reuter, M., Saueressig, F.: New J. Phys. 14, 055022 (2012). [arXiv:1202.2274 [hep-th]]

40. Ambjørn, J., Görlich, A., Jurkiewicz, J., Kreienbuehl, A., Loll, R.: Class. Quantum Gravity 31, 165003 (2014). [arXiv:1405.4585 [hep-th]]

41. Ambjørn, J., Jurkiewicz, J., Loll, R.: Phys. Rev. Lett. 95, 171301 (2005). [hep-th/0505113]

42. Coumbe, D.N., Jurkiewicz, J.: JHEP 1503, 151 (2015). [arXiv:1411.7712 [hep-th]]

43. Hořava, P.: Phys. Rev. Lett. 102, 161301 (2009). [arXiv:0902.3657 [hep-th]]

44. Lauscher, O., Reuter, M.: JHEP 0510, 050 (2005). [arXiv:hep-th/0508202]

45. Modesto, L.: Phys. Rev. D 86, 044005 (2012). [arXiv:1107.2403 [hep-th]]

46. Modesto, L.: Class. Quantum Gravity 26, 242002 (2009). [arXiv:0812.2214 [gr-qc]]

47. Calcagni, G., Oriti, D., Thürigen, J.: Phys. Rev. D 91, 084047 (2015). [arXiv:1412.8390 [hep-th]]

48. Carlip, S.: Class. Quantum Gravity 32, 232001 (2015). [arXiv:1506.08775 [gr-qc]]

49. Belenchia, A., Benincasa, D.M.T., Marcianò, A., Modesto, L.: Phys. Rev. D 93, 044017 (2016). [arXiv:1507.00330 [gr-qc]]

50. Calcagni, G.: Phys. Rev. D 86, 044021 (2012). [arXiv:1204.2550 [hep-th]]

51. Benedetti, D.: Phys. Rev. Lett. 102, 111303 (2009). [arXiv:0811.1396 [hep-th]]

52. Arzano, M., Trześniewski, T.: Phys. Rev. D 89, 124024 (2014). [arXiv:1404.4762 [hep-th]]

53. Arzano, M., Nettel, F.: Phys. Lett. B 767, 236 (2017). [arXiv:1611.10343 [hep-th]]

54. Mielczarek, J., Trześniewski, T.: Phys. Rev. D 96, 024012 (2017). [arXiv:1612.03894 [hep-th]]

55. Carlip, S.: Class. Quantum Gravity 34, 193001 (2017). [arXiv:1705.05417 [gr-qc]] 
56. Calcagni, G.: JHEP 1703, 138 (2017); 1706, 020 (2017) [arXiv:1612.05632 [hep-th]]

57. Sotiriou, T.P., Visser, M., Weinfurtner, S.: Phys. Rev. Lett. 107, 131303 (2011). [arXiv:1105.5646 [gr-qc]]

58. Calcagni, G., Eichhorn, A., Saueressig, F.: Phys. Rev. D 87, 124028 (2013). [arXiv:1304.7247 [hepth]]

59. Isham, C.J.: Proc. R. Soc. Lond. A 351, 209 (1976)

60. Calcagni, G.: Eur. Phys. J. C 76, 181 (2016); 76, 459 (2016) [arXiv:1602.01470 [hep-th]]

61. Bojowald, M., Brahma, S., Büyükçam, U., D’Ambrosio, F.: Phys. Rev. D 94, 104032 (2016). [arXiv:1610.08355 [gr-qc]]

62. Bojowald, M., Paily, G.M.: Phys. Rev. D 86, 104018 (2012). [arXiv:1112.1899 [gr-qc]]

63. Bojowald, M., Paily, G.M.: Phys. Rev. D 87, 044044 (2013). [arXiv:1212.4773 [gr-qc]]

64. Cailleteau, T., Mielczarek, J., Barrau, A., Grain, J.: Class. Quantum Gravity 29, 095010 (2012). [arXiv:1111.3535 [gr-qc]]

65. Cailleteau, T., Linsefors, L., Barrau, A.: Class. Quantum Gravity 31, 125011 (2014). [arXiv:1307.5238 [gr-qc]]

66. Bojowald, M.: Front. Phys. 3, 33 (2015). [arXiv:1409.3157 [gr-qc]]

67. Belinsky, V.A., Khalatnikov, I.M., Lifshitz, E.M.: Adv. Phys. 19, 525 (1970)

68. Belinsky, V.A., Khalatnikov, I.M., Lifshitz, E.M.: Adv. Phys. 31, 639 (1982)

69. Andersson, L., van Elst, H., Lim, W.C., Uggla, C.: Phys. Rev. Lett. 94, 051101 (2005). [arXiv:gr-qc/0402051]

70. Mielczarek, J.: Springer Proc. Phys. 157, 555 (2014). [arXiv:1207.4657 [gr-qc]]

71. Bojowald, M., Mielczarek, J.: JCAP 1508, 052 (2015). [arXiv:1503.09154 [gr-qc]]

72. Mielczarek, J.: AIP Conf. Proc. 1514, 81 (2012). [arXiv:1212.3527 [gr-qc]]

73. Mielczarek, J.: Europhys. Lett. 108, 40003 (2014). [arXiv:1304.2208 [gr-qc]]

74. Lévy-Leblond, J.-M., Ann, I.H.P.: Phys. Theor. 3, 1 (1965)

75. Amelino-Camelia, G., da Silva, M.M., Ronco, M., Cesarini, L., Lecian, O.M.: Phys. Rev. D 95, 024028 (2017). [arXiv:1605.00497 [gr-qc]]

76. Lukierski, J., Ruegg, H., Nowicki, A., Tolstoi, V.N.: Phys. Lett. B 264, 331 (1991)

77. Lukierski, J., Nowicki, A., Ruegg, H.: Phys. Lett. B 293, 344 (1992)

78. Magueijo, J., Smolin, L.: Phys. Rev. Lett. 88, 190403 (2002). [arXiv:hep-th/0112090]

79. Amelino-Camelia, G., Freidel, L., Kowalski-Glikman, J., Smolin, L.: Gen. Relativ. Gravit. 43, 2547 (2011). [arXiv:1101.0931 [hep-th]]

80. Calcagni, G., Ronco, M.: Phys. Rev. D 95, 045001 (2017). [arXiv:1608.01667 [hep-th]]

81. Born, M.: Proc. R. Soc. Lond. A 165, 291 (1938)

82. Snyder, H.S.: Phys. Rev. 71, 38 (1947)

83. Majid, S.: Foundations of Quantum Group Theory. Cambridge University Press, Cambridge (1995)

84. Majid, S., Ruegg, H.: Phys. Lett. B 334, 348 (1994). [arXiv:hep-th/9405107]

85. Kowalski-Glikman, J.: Phys. Lett. B 547, 291 (2002). [arXiv:hep-th/0207279]

86. Kowalski-Glikman, J., Nowak, S.: Class. Quantum Gravity 20, 4799 (2003). [arXiv:hep-th/0304101]

87. Amelino-Camelia, G.: Int. J. Mod. Phys. D 11, 35 (2002). [arXiv:gr-qc/0012051]

88. Amelino-Camelia, G.: Nature 418, 34 (2002). [arXiv:gr-qc/0207049]

89. Girelli, F., Livine, E.R., Oriti, D.: Nucl. Phys. B 708, 411 (2005). [arXiv:gr-qc/0406100]

90. Amelino-Camelia, G., Freidel, L., Kowalski-Glikman, J., Smolin, L.: Phys. Rev. D 84, 084010 (2011). [arXiv:1106.0313 [hep-th]]

91. Cianfrani, F., Kowalski-Glikman, J., Rosati, G.: Phys. Rev. D 89, 044039 (2014). [arXiv:1401.2057 [gr-qc]]

92. Barcaroli, L., Brunkhorst, L.K., Gubitosi, G., Loret, N., Pfeifer, C.: Phys. Rev. D 92, 084053 (2015). [arXiv:1507.00922 [gr-qc]]

93. Freidel, L., Leigh, R.G., Minic, D.: Phys. Lett. B 730, 302 (2014). [arXiv:1307.7080 [hep-th]]

94. Freidel, L., Leigh, R.G., Minic, D.: JHEP 06, 006 (2015). [arXiv:1502.08005 [hep-th]]

95. Oriti, D.: In: Murugan, J., Weltmann, A., Ellis, G.F.R. (eds.) Foundations of Space and Time: Reflections on Quantum Gravity. Cambridge University Press, (2012). [arXiv:1110.5606 [hep-th]]

96. Girelli, F., Livine, E.R., Oriti, D.: Phys. Rev. D 81, 024015 (2010). [arXiv:0903.3475 [gr-qc]]

97. Bojowald, M., Kempf, A.: Phys. Rev. D 86, 085017 (2012). [arXiv:1112.0994 [hep-th]]

98. Mielczarek, J., Trześniewski, T.: Phys. Lett. B 759, 424 (2016). [arXiv:1601.04515 [hep-th]]

99. Mielczarek, J.: Universe 3, 29 (2017). [arXiv:1612.04355 [hep-th]]

100. Trześniewski, T.: Acta Phys. Pol. B Proc. Suppl. 10, 329 (2017). [arXiv:1701.06865 [hep-th]] 
101. Bilski, J., Brahma, S., Marcianò, A., Mielczarek, J.: arXiv:1708.03207 [hep-th]

102. Born, M., Infeld, L.: Proc. R. Soc. A 144, 425 (1934)

103. Mielczarek, J., Trześniewski, T.: Phys. Rev. D 96, 043522 (2017). [arXiv:1704.01934 [gr-qc]]

104. Ashtekar, A.: Phys. Rev. Lett. 57, 2244 (1986)

105. Rovelli, C., Smolin, L.: Nucl. Phys. B 331, 80 (1990)

106. Bahr, B., Dittrich, B., Geiller, M.: arXiv:1506.08571 [gr-qc]

107. Rovelli, C., Vidotto, F.: Phys. Rev. D 91, 084037 (2015). [arXiv:1502.00278 [gr-qc]]

108. Dittrich, B.: JHEP 1705, 123 (2017). [arXiv:1701.02037 [hep-th]]

109. Dittrich, B., Geiller, M.: New J. Phys. 19, 013003 (2017). [arXiv:1604.05195 [hep-th]]

110. Riello, A.: Phys. Rev. D 97, 025003 (2018). [arXiv:1706.07811 [hep-th]] 